\title{
"The tonic's not always in a bottle": a qualitative study investigating a heart rate variability biofeedback coherence intervention for individuals with gastrointestinal disorders
}

\author{
Lucy Field ${ }^{1}$, Mark Forshaw ${ }^{2}$, Helen Poole ${ }^{3}$, Peter Paine ${ }^{4}$, Gillian Fairclough ${ }^{5}$, \\ Candice Walton ${ }^{6}$ \\ ${ }^{1,2,3}$ Liverpool John Moores University, School of Psychology, Faculty of Health, Tom Reilly Building \\ Byrom Street, Liverpool, L3 3AF, UK \\ ${ }^{4}$ Department of Gastroenterology, Salford Royal Hospital, Stott Lane, Salford M6 8HD, England, UK \\ ${ }^{4}$ Centre for GI Sciences, Division of Diabetes, Endocrinology and Gastroenterology, Faculty of Biology, \\ Medicine and Health, University of Manchester, Manchester M13 9PL, UK \\ ${ }^{5,6}$ Department of Clinical Health Psychology, Salford Royal Hospital, \\ Stott Lane, Salford M6 8HD, England, UK \\ ${ }^{1}$ Corresponding author \\ E-mail: ${ }^{1}$ lucyfield2222@gmail.com, ${ }^{2}$ m.j.forshaw@ljmu.ac.uk, ${ }^{3}$ h.m.poole@ljmu.ac.uk, \\ ${ }^{4}$ peter.paine@srft.nhs.uk, ${ }^{5}$ gillian.fairclough@elht.nhs.uk, ${ }^{6}$ Candice.Walton@srft.nhs.uk
}

Received 20 May 2021; received in revised form 9 September 2021; accepted 3 November 2021 DOI https://doi.org/10.21595/chs.2021.22069

Check for updates

Copyright (C) 2022 Lucy Field, et al. This is an open access article distributed under the Creative Commons Attribution License, which permits unrestricted use, distribution, and reproduction in any medium, provided the original work is properly cited.

\begin{abstract}
Gastrointestinal disorders (GID) affect psychological and physical health and can be impacted by stress and ability to self-regulate emotions. The aim of this study was to gain an indepth understanding of individuals with GID experiences of a Heart Rate Variability biofeedback (HRV-BF) coherence intervention. Six patients, who had already been diagnosed with GID, were interviewed using a semi-structured interview schedule to investigate how engaging in an HRVBF coherence intervention affected their levels of stress and their GID condition. All transcripts were explored using Interpretative Phenomenological Analysis (IPA). Analysis of the accounts indicated four superordinate themes: (1) Autonomy, (2) Self-regulation, (3) Acceptance, (4) Mind-Body-Spirit. The field of HRV-BF interventions in the area of gastroenterology is promising. However, it is recommended that further studies involving psycho-physiological interventions, with mixed-method designs and larger samples, are undertaken.
\end{abstract}

Keywords: heart rate variability, gastrointestinal disorders, HeartMath, coherence, heart rhythms.

\section{Introduction}

Gastrointestinal disorders (GID) negatively affect quality of life and psychological health [1]. Furthermore, there are significant economic impacts on health care systems and secondary costs from absenteeism and impaired productivity [2]. GID disorders can be either organic (e.g. Inflammatory bowel disease (IBD), including Crohn's disease and Ulcerative Colitis) or functional (e.g. Irritable Bowel Syndrome (IBS) and constipation). However, presentation is not always clear cut.

Healthcare, as it stands, is principally developed on a reactive disease treatment model and a move towards a proactive health-enhancement model is to be transformational. More recently, there has been a surge of interest in mind-body medicine, not only by clinicians but also by patients [3]. Greater involvement of patients in their own care is critical, and it is vital that individuals understand their chronic disease and how they can reduce symptoms and alleviate distress [4].

During the last few years, there has been a move in the Gastrointestinal field towards a biopsychosocial model. Dysregulation of the brain-gut axis is the aim of treatment for GIDs with the awareness that patients' symptoms are the consequence of a dynamic between biomedical, gastrointestinal and psychosocial processes [4]. There are a wide range of treatments available, 
including dietary therapies, behavioural and psychological therapies and drug treatments which have all been well recognised and validated in controlled trials [2]. In a recent review gut-directed hypnotherapy, E-treatment/telemedicine, Cognitive Behavioural Therapy and Biofeedback assisted relaxation training, were all found to be effective in treating GIDs, by supporting adaptive coping, providing symptom relief, and improving quality of life [4]

One area that has become apparent in GIDs is the impact of stress. It has been shown that chronic episodes of stress may negatively impact the Autonomic Nervous System (ANS). This leads to the release of large quantities of stress hormones, such as glucocorticoids (e.g. cortisol) and catecholamines (e.g. norepinephrine and epinephrine) [5]. These hormones have a direct effect on the Enteric Nervous System (ENS) and can cause detrimental changes in visceral sensitivity and gastrointestinal motility [6]. Stressful events typically affect respiration, blood pressure, and immune system functioning; acute and chronic stress increases allostatic load in the body which over time causes mental and/or physical health problems [7]. Therefore, stress management interventions in this population group are critical.

This research focuses on an intervention aimed at improving self-regulation of a key aspect of the stress response, Heart Rate Variability (HRV) biofeedback. HRV is a measure of beat-to-beat variations in heartbeat, often called cardio-vagal tone [7]. The heart produces the strongest electrical signal in the body and, during coherence, the heart rhythm oscillates at a frequency of $0.1 \mathrm{~Hz}$ oscillations. Brainwaves can synchronise to $0.1 \mathrm{~Hz}$ oscillations in areas of the brain such as the prefrontal cortex $[8,9]$. The vagus nerve is the tenth, longest cranial nerve ranging from the brainstem to the colon and is the predominant neural substrate of the parasympathetic nervous system [10]. This nerve has both efferent and afferent pathways and has a critical role in gastrointestinal motility, information and pain. The functioning of the nervous system can be enhanced by improving the state of HRV coherence in the body. As this occurs, there is increased activity in the prefrontal cortex and a reduction in stress; consequently, this can enhance ability to self-regulate thoughts, feelings and behaviours [11].

Coherence can be optimised by regulating breathing and by intentionally experiencing positive and pleasant emotions which have been found to benefit physical and mental health and quality of life [12]. Six breaths per minute respiratory rate is an effective way to create coherence within the autonomic nervous system and between heart and brain [8]. HeartMath technology uses biofeedback (BF) principles to teach individuals about their HRV coherence in real-time and facilitate an understanding of the nervous system [13]. It is proposed that the key to management of stress is firstly to become aware of it and, secondly, be able to self-regulate the emotional response.

Studies over recent years have found a $75 \%$ accuracy in determining emotional quality from examining HRV coherence patterns [7]. Negative emotions such as anger and frustration, create jagged and incoherent heart rhythm patterns which send incoherent signals to the brain. On the other hand, positive emotions such as love, care and compassion create coherence as demonstrated by a smooth and sine-like wave pattern [7]. Becoming coherent can be initiated by regulating the breath and increased by intentionally experiencing positive and pleasant emotions which have been found to have profound benefits on physical, mental health and quality of life [12].

Of particular interest in this research are interventions that have used HeartMath Technology to measure HRV coherence. HeartMath technology uses biofeedback principles to teach individuals about their heart rate variability coherence in real-time and facilitate an understanding of the nervous system. It is proposed that the key to management of stress is firstly to become aware of it and, secondly, be able to self-regulate the emotional response.

The ability to self-regulate emotions and feelings have a strong and powerful effect on our heart-brain connection and entrain the flow of information throughout the psycho-physiological network [7]. This bottom-up approach, from the heart to the brain, directly alters physiology and produces more coherent systems which improve an individual's ability to self-regulate and consequently improve health and performance [12]. Of key significance for this research is that scientists at the forefront in this field propose that it is the state of our physiological and nervous 
system that regulates the stability and quality of the emotions we experience [7].

A recent systematic review concluded that HRV is an objective measure and beneficial tool in categorising function of the ANS [14]. A number of studies have found that low HRV patterns are associated with reduced health and morbidity in comparison with high HRV readings which indicate improved mental and physical health [15-17]. A large and growing body of evidence has shown HRV-BF interventions improve levels of HRV for a range of mental and physical health problems $[18,19]$.

There are a growing number of quantitative research studies around the effects on HRV-BF intervention in individuals with GIDs which are beyond the scope of this review (for more information, please refer to $[20,21]$. In more recent years, research has started to examine the qualitative component regarding HRV-BF interventions within the general populations that have found these interventions to be effective in reducing stress, improving mood and developing coherence $[22,23]$. A recent study [24] implemented a HeartMath training protocol with Olympic athletes and analysed the data using thematic analysis. They found themes of improved ability to self-regulate both physiological and psychological states and an enhanced sense of personal control following the intervention. A mixed-method study investigated functional gastrointestinal disorders with 27 paediatric individuals who attended between 3-18 HRV-BF sessions [25]. The researchers found improved quantitative measures on clinical outcome measures of IBS and FAP but, what was most interesting for the purpose of this research, was the qualitative report. Participants reported feeling validated in their symptoms and felt "believed" that the condition was not "all in their head". Participants reported showing an interest in the psychophysiology of their body and reinforced that the intervention was effective for symptom reduction and patient engagement. However, as no specific type of qualitative design was employed, there were no themes identified in the data. Therefore, it was unclear what the exact procedure was; hence the qualitative analysis is limited. There is very little qualitative research implemented in HRV-BF studies with individuals with GID.

There is, however, research that highlights the difficulties individuals with GID face. In a qualitative study researchers explored the lived experiences of individuals with Crohn's disease (CD) and found they worry about symptom management, such as diarrhoea and constant pain and that disrupts leisure activities, social lives and work [26]. This research found that individuals with $\mathrm{CD}$ had increased risks of anxiety and depression. Therefore, the literature highlights the importance of dealing with some of these preoccupations to see whether particular interventions improve lived experiences of condition management.

Several studies investigating individuals with IBS have found that they have erratic HRV patterns when compared to a control group, and this has a greater impact in women than men [6]. These findings suggest individuals with GID may have ANS dysregulation; hence enhancements in HRV coherence are anticipated to make advantageous changes in the ENS [20].

\section{Rationale}

Further qualitative studies are required to understand the individual experiences of using HRV-BF coherence interventions and its effects on perceptions of emotional states [27-29] especially within individuals who have GID. It is proposed that some GID symptoms may be associated with incoherent heart rhythm patterns caused by stress and that by educating individuals to self-regulate their heart rhythm patterns at a frequency of 0.1 oscillations then HRV coherence will be improved. It is theorised that as a result, GI symptoms may be reduced and psychological outcomes improved [30]. This could pave the way to develop effective, evidenced-based HRV-BF interventions that are cost-effective and patient-centred. 


\section{Methods}

\subsection{Participants}

Six participants (age range 18-60) were recruited from the Gastroenterology and Clinical Health Psychology Department at a hospital in the north of England. All patients were assessed as having either a functional or organic GID and were wait-listed for treatment.

This sample size was appropriate for using an Interpretative Phenomenological Analysis (IPA) approach (see details below) as it is within the recommended boundaries related to size [31]. Studies using IPA generally have sample sizes between 1 and 9 [32].

\subsection{Inclusion criteria}

Individuals were included if they were over the age of 18 and declared no major physical disorder (other than GID) or severe mental disorder. Suitability for the study was assessed by a Consultant Gastroenterologist and a Clinical Psychologist. Patients could only take part if they had a 'smartphone' due to technical limitations of the intervention.

\subsection{Exclusion criteria}

Participants who were awaiting surgery or had any other major physical or mental disorder were excluded, as were individuals who did not have sufficient understanding of English.

\subsection{Design}

Participants were invited to take part in a six-week HRV-BF coherence intervention that consisted of educational components of the mind-body connection and relationship to stress, and extensive HRV-BF coherence practice with weekly feedback from the researcher. At the end of the intervention, participants were interviewed about their experience of using the HeartMath HRV-BF coherence equipment, stress and their gastrointestinal symptoms (See Appendix for interview schedule).

\subsection{Apparatus}

The researchers utilised equipment from the HeartMath Institute, including the Inner Balance ${ }^{\circledR}$ bluetooth sensor and the emWave ${ }^{\circledR}$ Pro Plus.

Inner balance: The Inner Balance ${ }^{\circledR}$ sensor for Android and iPhone is an innovative approach to improving wellness through monitoring and self-regulation of thoughts, feelings and physiology. Participants were required to connect the sensor to their smart-phone via the app and connect the ear clip to the ear lobe. Participants were then instructed to follow the breathing pacer to help moderate breathing and to activate a positive, renewing emotion. The Inner Balance app. provides 'real-time' feedback on their HRV coherence and techniques to create more coherent heart rhythms. Each participant was given an sensor for home coherence practice.

emWave ${ }^{\circledR}$ Pro Plus: The emWave ${ }^{\circledR}$ Pro Plus consists of PC based software and a USB sensory unit and ear sensor that measures HRV coherence and can produce assessments pre-post interventions. This apparatus was purchased through the UK HeartMath website, http://heartmath.co.uk/.

\subsection{Intervention}

All patients submitted their signed consent form and an information sheet before the commencement of any research. Participants were informed of the session format over the six weeks and were sent a zoom link (for online participants) or an appointment time (for face to face 
appointment prior to COVID-19) with the same time slot each week. All participants were provided with a Inner Balance ${ }^{\circledR}$ Bluetooth sensor and given instruction on how to use. All sessions were 50 minutes long.

For the first session, participants were given an introduction into the HeartMath technology, the plan of expected sessions and a time to ask any questions. For session two participants were shown 'the quick coherence technique' and three stages of 'effective coherence training'. Individuals were educated about their own physiology via the involuntary HRV and connection to mind and taught how to reach an optimal HRV state. Participants were asked to practice three-five-minute sessions every day at home with their inner balance.

In session three participants were taught the four domains of resilience (physical, emotional, mental, spiritual) and explored energy draining and renewing activities [13]. Session four investigated the depletion to renewal energy grid and individuals were taught the 'heart lock in technique' and practiced HRV coherence training with guidance from the researcher. For session five, participants were guided through the 'freeze frame technique' and practiced HRV coherence. For the final session, participants were asked to complete an interview on their experiences of taking part in the research.

\subsection{Interview}

Each interview lasted between 13 and 29 minutes and was recorded and transcribed. Participants were debriefed at the end of the study.

Ethical approval was gained from NHS Research Ethics Committee, IRAS reference number 250048, and the research team at Liverpool John Moores University.

\subsection{Data analysis}

Qualitative research provides an individual patient perspective which is critical to ensure healthcare services are patient-centred. Interpretative Phenomenological Analysis (IPA) is used to gain a deeper understanding of an individual's lived experiences, and how they construct sense from these experiences [31]. Using this qualitative approach, a double hermeneutic is generated as the researcher is trying to make sense of the participants' interpretations. The analysis involves three steps: 1) understanding content 2) examining use of language, 3) interacting with the data deeply and conceptually.

Any patterns and relationships within the pieces of data were noted individually, and then collectively for all participants. This enabled themes to be generated, which were then re-examined in the interview texts. Line-by-line analysis was completed, which led to development of key themes. These were re-checked to ensure the interpretation was grounded in the original transcript [31].

Reflexivity was implemented throughout the analytical process. The authors were aware of their lived experiences, clinical encounters and personal beliefs which may influence the analysis [26].

\section{Results}

The analysis of the results revealed four main Superordinate themes: autonomy, selfregulation, acceptance and mind-body-spirit. These are displayed in Table 1.

\subsection{Autonomy}

This theme comprised three subthemes: powerlessness, taking back control and regeneration which were consistent throughout the data. 
“THE TONIC'S NOT ALWAYS IN A BOTTLE”..

Lucy Field, Mark Forshaw, Helen Poole, Peter Paine, Gillian Fairclough, Candice Walton

Table 1. Superordinate and subordinate themes

\begin{tabular}{|c|c|}
\hline Superordinate theme & Subordinate theme \\
\hline \multirow{2}{*}{ Autonomy } & Powerlessness \\
\cline { 2 - 2 } & Taking back control \\
\cline { 2 - 2 } Self-regulation & Regeneration \\
\cline { 2 - 2 } Acceptance & Awareness \\
\hline \multirow{2}{*}{ Mind-Body-Spirit } & Connectedness \\
& $\begin{array}{c}\text { Self-compassion } \\
\text { Mental and emotional benefits } \\
\text { Physimportance of self } \\
\text { Self as healer }\end{array}$ \\
\hline
\end{tabular}

\subsubsection{Powerlessness}

The first subtheme was chosen as patients spoke about feeling helpless within their life because of their condition: Ross stated "I've felt I've had no control in my life because of the ill health" and Janet revealed "When you feel no control, you feel vulnerable, fully exposed."

One of the patients, Izzy (who had a colostomy and ileostomy bag fitted after surgery), explained how her lack of control led to her feeling that her body was owned by the medical profession: "I speak on IBD forums with other people who feel like the body is just owned by the medical profession, you know they have no control over when they are ill, how they can try and feel better, having a life they just feel they exist." Izzy compared the trauma from the surgery as a sense of invasion "my body has been invaded for so long, on so many levels."

\subsubsection{Taking back control}

The second subtheme became evident as participants spoke about the benefits of being able to gain some control of their body following the HRV-BF sessions. James reported "It's giving me some control" and John remarked "I now have a better understanding of the impact that my body has with the stress levels. I have to try and control it."

There was a sense that patients had learned how to identify stress in the body and consequently could choose to moderate it using HRV-BF, and in some cases, reduce pain. Janet reported: "I control to a point how stressed my body is and how relaxed it can be, and by taking control and listening to my body, I'm putting it in a more harmonious state so that it is not constantly in pain and just having a detrimental effect on the whole of my day and my outlook basically with life."

The use of HRV coherence appeared to be helpful for patients to understand their physiology and their own internal states, as shared by Ross: "I think in this case with the heart monitor, so many people will benefit from this technique and just taking that control back would help them massively mentally and physically and that for me, just sums up my experience of this study."

\subsubsection{Regeneration}

The third subtheme arose from the newness that many patients reported to feel following the intervention. Sarah testified that using the techniques and principles in her life gave her a new lease of life: "I'm living life again and not just existing."

Ross reflected on his increased confidence in engaging in hobbies and new experiences that he had given up on: "I feel like with this I can make plans rather than feeling like I can't do anything, as I will have pain all day and there will be accidents. And it's not happened like that. It's put me in a mindset where I thought. No! I'm doing this today; I'm doing it!'

Izzy shared a similar sense of empowerment and recognition which was welcomed and embraced as a journey back to her life: "I just feel this has given me a life-line that nobody else has offered me in over 10 years." 


\subsection{Self-regulation}

Self-regulation comprised two subthemes: awareness and connectedness.

\subsubsection{Awareness}

This subtheme was chosen as all patients reported an increase in their knowledge after completing the HRV-BF sessions. Janet stated: "The sessions have been really helpful, and enlightening," and Izzy reported, "It educated me." In addition, patients described that they became more aware of themselves.

John reflected: "I found it very useful in the sense that it gives me an opportunity to engage with my own thoughts and having my own space." Ross stated: "It makes you realise what the triggers are and how you can deal with them differently."

Patients revealed that they had never been taught about their own body, or how to self-regulate. Instead, they had previously relied on other people or taken more medication to manage symptoms. It appeared that a sense of empowerment, ownership and confidence was developed as patients used the HRV-BF principles to self-regulate as reflected by Izzy: "I can say, Hang on a minute! I can just do my breathing. I can bring this back around myself rather than rely on someone else to be helping me ... or ... and I suppose for me it's been very worthwhile." The sense of pausing and stopping, to re-connect with their own bodies and implanting their learning, appeared to help manage stress. Ross shared: "I found that I know my stress levels are attributing to a lot of problems I am having, so now I'm stopping and checking myself, it's a learning process still."

\subsubsection{Connectedness}

The second subtheme was developed as all patients spoke of the realisation that their mind and body were entwined, and this was demonstrated through increased practice and understanding of HRV-BF training. Janet conveyed: "I learnt there is a big connection between your breathing and heart rate ... erm ... and how that affects stress levels and that it is fairly easy to control with practice." And Izzy stated: "every time I've done the breathing, I've felt less stressed and there is definitely a direct connection between stress and symptoms. I'd say the training has had a positive effect."

The connection between thoughts, breathing and heart rate variability coherence was clearly evidenced. Ross reflected that "I think it was the initial learning that everything is so interconnected, erm ... that realisation was probably the most important thing. I hadn't realised the extent that your breathing and thought processes can have such a serious effect on your HRV." Additionally, Sarah explained: "I learnt that your thoughts and feelings are very powerful, and your pulse reacts to your thoughts and there is no getting away from that as demonstrated by the technology."

It was clear that patients had not just connected with a theoretical basis for understanding the HRV-BF training but really felt the experience of coherence and embodied the practice. John explained: "you can change your thoughts and feelings by using the techniques to channel yourself to be more co-ordinated in your body." Patients appreciated someone to talk to and the use of real time of heart rhythm patterns appeared to be very helpful. Ross expressed: "I suppose it's nice to talk to someone about your thoughts and feelings and seeing the evidence in front of you from an interactive point of view;" and more interestingly recognised that "you can't lie to it... it will tell you are in a state of incoherence, and it will confront you with where you are."

\subsection{Acceptance}

Acceptance comprised two subthemes self-compassion and recognition of importance of self. 


\subsubsection{Self-compassion}

Following the intervention, all patients developed more care and kindness for themselves. Sarah qualified this: "By giving my body more time, looking after myself better, rather than just hating my body and beating myself up, I've learned acceptance, and just by looking after myself in a better way and taking time out not feeling guilty which has always been a big thing for me." Feeling guilty and being hard on ones self was present with the patients regularly. Janet detailed: "I've learnt being fairer to myself and 'more realistic. It makes it easier to manage, being aware and doing things that make you feel good, renewing, you know planning to do something nice, you know something simple, like going for a walk." In a similar vein John reflected: "It is acceptable to feel upset or down and to struggle, but it's about how you can change, by using the techniques. I feel a lot more content. So, in that sense, it's a no brainer that I have never thought of it in that way before."

\subsubsection{Recognition of importance of self}

Towards the end of the six-week intervention, patients realised that they were important and deserving of self-care and time and appreciated how this linked to their health. John reflected: "The most helpful thing I realised is how I was impacting on my own health... and burning myself out... ...putting work first and myself last in everything I do;" and Ross realised "I am easier on myself. And erm... it's got to have a positive impact on my condition."

Within this, it appeared that a sense of permission was embedded. As Janet suggested: "I realised that I didn't put 'me' anywhere near where I should be... you know time for me was always time for everyone else. I realised I can allocate me time, no matter what. I think I need to give myself a little bit of credit and to look after myself instead of everyone else."

There was an apparent appreciation that individuals no longer needed to feel guilty about having "time out" for rest, and were confident about their desire to do so. Izzy signalled: "I just take time out for myself but not feel guilty for having to do that, not feeling I have to explain myself to people why I am at home and perhaps not back in work yet."

\subsection{Mind-body-spirit}

This theme had three subordinate themes: physical benefits, mental and emotional benefits and self as healer.

\subsubsection{Physical benefits}

HRV-BF training appeared to be effective for pain management. John indicated: "I've certainly not had the degree of pain for so long as what I was having before." Izzy indicated other physical changes: "My ileostomy ... since I have been doing this study for me ... the output ... it's not bled like it used to do, for me when I was stressed it used to bleed when I was having pain and I was feeling anxious ... my abdomen was very rigid and the pain ... erm ... at times would be very overwhelming, with this ... I believe I feel within myself that I have not had the pain as much that I have needed the medication I have gone into the breathing, straight away... I'm starting to relax and the pain dissipates. So for me, I know this has been key as it has been able to help me reduce the medication."

Sarah often experienced urgency in using the toilet: "I was stressed out and I went to bed and did some breathing which I don't normally do ... erm now normally without being too detailed I can't get to the toilet quick enough, you know, and I've been like that for a long time now and for the first time I wasn't like that. And it was only when I stopped and thought about it and thought, I did the breathing last night and wondered if it had contributed to this. So, I need to do more of that and see what happens, whether it was a fluke or a one off, but I don't seem to have as many 
troubles with my stomach I must admit."

Sarah concluded that "no one would get me to change but I am seeing the impact it's having on me and my body... it's all a benefit isn't it?"

\subsubsection{Mental and emotional benefits}

There was change after periods of coherence practice in participants reported mental and emotional states. Ross revealed: "It just felt really calming like a load had been lifted... yes that feeling of a reset, feeling fresh, it makes you feel immediately calm afterwards;" and Sarah mirrored: "It's relaxing ... makes me feel more content." John identified: "There are benefits to myself; ... I feel better myself doing things that are renewing for me and recharge my batteries and all the things make me feel better."

Janet explored the mental aspect of having a gastrointestinal condition: "Your mind doesn't rest because the gastro problem is there constant and the more uptight you are the worst your stomach is. Erm... the techniques help calm that pathway down, so I wasn't as bad, the breathing helped when I was having a really bad day."

Reflecting on the overall experience of the HRV-BF intervention, Izzy concluded: "The takeaway for me is that without this, I would be in a worse place than where I am now, I am in a much better place, I am in a stronger place, is the key word for me ...mentally and physically."

\subsubsection{Self as healer}

Many of the patients spoke about having surgery or medical procedures as a result of their gastrointestinal symptoms. Janet shared her experience of looking at her physical health condition differently following the intervention: "The tonic's not always in a bottle, it can be mental, emotional, by taking time out for my body, not feeling that I'm being lazy; by lying there on a sofa doing the breathing, doing the exercises thinking of positive things, that's not me being lazy; that's me taking time out because I need to give it something back. It's like my body's cry for help." This empowering voice led to the sense that she had taken charge of her own body and cultivated an environment for healing, by bringing her own heart rhythm patterns into coherence.

\section{Discussion}

The results of this qualitative six-week HRV-BF coherence study showed favourable outcomes for individuals with GID. IPA identified four main Superordinate themes: autonomy, selfregulation, acceptance and mind-body-spirit.

Under the theme Autonomy, participants reported feeling vulnerable and overwhelmed by their health conditions, particularly around the lack of somatic control. Feeling out of control is a reoccurring theme in the GID literature. Individuals with GI symptoms have reported that their daily living felt compromised and they were controlled by their condition, particularly in relation to going to work or social events, due to preoccupation with being close to toilet facilities [26]. Patients with IBD reported inability to control bodily functions as the most distressing part of living with their condition [33]. This study showed HRV-BF coherence improved participants control and understanding over both their mind and body. Individuals became aware of how they could intentionally activate self-regulation techniques and implement them for symptom management relief in real-time.

Under the theme Self-regulation, a sense of ownership was cultivated through sessions, as an effective way of managing stress. Participants recognised that entering into a state of coherence was not an abstract concept but a reality, and one that all participants were able to cultivate and feel. These experiences are consistently reported in the literature [28, 29].

Under the theme, Acceptance, individuals recognised that cultivating kindness for themselves was not only beneficial for their mind but also for their body and quality of life. At the start of the 
intervention, participants spoke about guilt and feeling responsible for their health conditions. Acceptance has been identified as important in the IBD literature particularly around adjustment to living with a stoma and a long-term health condition [33]. Self-compassion has been linked to improved gastrointestinal tract motility and immune function [34]. Participants can be taught self-compassion which is beneficial for individuals who feel guilty or blame themselves for their GID $[35,36]$.

Under the Mind-Body-Spirit theme, some patients reported a reduction in pain. All clients noticed an improvement in their gastrointestinal symptoms following the intervention. One participant described the intervention as "The tonic's not always in a bottle", with the recognition that psychological components of healing can help physical aspects. One patient even noticed reduced stoma output and bleeding following HRV-BF coherence practice. One previous case report of HRV-BF after ileostomy formation reported psychological but not stomal improvements [37]. Furthermore, individuals reported feeling more relaxed, calmer, and renewed following the HRV-BF training, in keeping with previous studies [29].

As a whole, the themes provide evidence HRV-BF coherence interventions raise awareness of both the psychological and physiological components of stress, with beneficial effects on mind-body health. The importance of self-control within individuals with GIDs is reiterated throughout the literature [38]. Resilience, self-regulation, and optimism are three individual strengths in the context of psychogastroenterology that have strong associations with symptom experience in other chronic disease groups [39]. Optimism has been linked to healthier autonomic function and increased levels of HRV [40] which are increasingly implicated in the pathophysiology of gastrointestinal disorders [41-43]. Future HRV studies could incorporate measures of optimism and resilience within their study design. It is clear that all participants were able to achieve a state of coherence which was a new health behaviour for them; the mastery of this technique improved self-confidence and perspective taking, which are directly linked to positive health outcomes [44].

This study provides detailed insight into the experiences of patients with functional and organic GIDs and suggests that by empowering individuals to self-regulate their emotions, they are gaining some level of control. Further, this research shows that HRV-BF interventions are amenable to digital delivery. The rise in digital technology and wearable sensors has grown dramatically in recent years, with the general population interested in lifestyle apps for health (e.g. sleep trackers or fitness trackers). This domain is gaining traction and it is proposed that HRV-BF devices, and diaphragmatic breathing apps, could be implemented in the teaching of disease self-management skills as part of a patient's care and may be administered remotely [45-47].

\section{Limitations}

Only six participants were included in the study, reducing generalisability. Yet, the results suggest positive effects across genders. Furthermore, this study followed recommendations to ensure strong validity throughout the research process [48]. The researcher demonstrated sensitivity to context by understanding participants' viewpoints, linguistic perceptions, and the socio-culture within which the research was conducted. These factors were considered salient as they may influence not only how the participant reports, but also what they report and how that is interpreted by the researcher. The themes generated throughout the data were sensitively and carefully derived rather than by imposing pre-determined categories on the data to ensure commitment and transparency of the approach. The present findings support previous qualitative literature in biofeedback studies that have found increased awareness, improved self-regulatory capacity and adaptive behavioural change following a HRV-BF intervention [49]. Collectively, both the present study, and existing literature, highlight that biofeedback interventions, using HeartMath technology, reveal greater self-regulation, enhanced autotomy, enriched acceptance and a better understanding of the Mind-Body-Spirit [28, 29]. They also extend previous findings to show improved benefit for individuals with either a functional or organic GID. Further studies 
involving psycho-physiological interventions, with mixed-method designs and using larger samples, should be undertaken, that also include a detailed HRV analysis [29]. Future studies will also need to consider what represents optimal dosing of HRV coherence practice.

\section{Conclusions}

The study findings support an integrative approach to wellness and ill-health that targets mental, emotional, physical, and spiritual aspects of the whole person [50]. It is important that these types of research studies are developed in order to understand not only how best to personalise such methodologies that maximise public health potential, but also are easy for patients to learn, non-invasive and without known side effects [3, 37].

This study has specifically shown the potential for HRV-BF coherence as a self-management tool to improve symptoms in gastrointestinal conditions. Given the widespread availability of apps and devices to monitor heart rate and the lack of evidence for adverse effects, this type of intervention provides a low-cost option for patients to try. It follows that this could also demonstrate to what extent these practices can shift the course of disease and minimise the need for pharmaceuticals and invasive tests and procedures.

\section{References}

[1] A. D. Sperber et al., "Worldwide Prevalence and Burden of Functional Gastrointestinal Disorders, Results of Rome Foundation Global Study," Gastroenterology, Vol. 160, No. 1, pp. 99-114.e3, Jan. 2021, https://doi.org/10.1053/j.gastro.2020.04.014

[2] C. Basnayake, M. A. Kamm, M. R. Salzberg, A. Wilson-O. 'Brien, A. Stanley, and A. J. Thompson, "Delivery of care for functional gastrointestinal disorders: A systematic review," Journal of Gastroenterology and Hepatology, Vol. 35, No. 2, pp. 204-210, Feb. 2020, https://doi.org/10.1111/jgh.14830

[3] M. L. Dossett, G. L. Fricchione, and H. Benson, "A new era for mind-body medicine," New England Journal of Medicine, Vol. 382, No. 15, pp. 1390-1391, Apr. 2020, https://doi.org/10.1056/nejmp1917461

[4] B. Reed, J. Buzenski, and M. A. L. van Tilburg, "Implementing psychological therapies for gastrointestinal disorders in pediatrics," Expert Review of Gastroenterology and Hepatology, Vol. 14, No. 11, pp. 1061-1067, Nov. 2020, https://doi.org/10.1080/17474124.2020.1806055

[5] J. K. Mogilski, A. Wysocki, S. D. Reeve, V. E. Mitchell, J. Lunge, and L. L. M. Welling, "Stress hormones, physiology, and behavior," in The Oxford Handbook of Evolutionary Psychology and Behavioral Endocrinology, Oxford University Press, 2019, pp. 350-365, https://doi.org/10.1093/oxfordhb/9780190649739.013.19

[6] W. S. Waring, M. Chui, A. Japp, E. F. Nicol, and M. J. Ford, "Autonomic cardiovascular responses are impaired in women with irritable bowel syndrome," Journal of Clinical Gastroenterology, Vol. 38, No. 8, pp. 658-663, Sep. 2004, https://doi.org/10.1097/01.mcg.0000135362.35665.49

[7] J. Elbers and R. McCraty, "HeartMath approach to self-regulation and psychosocial well-being," Journal of Psychology in Africa, Vol. 30, No. 1, pp. 69-79, Feb. 2020, https://doi.org/10.1080/14330237.2020.1712797

[8] A. R. Schwerdtfeger, G. Schwarz, K. Pfurtscheller, J. F. Thayer, M. N. Jarczok, and G. Pfurtscheller, "Heart rate variability (HRV): From brain death to resonance breathing at 6 breaths per minute," Clinical Neurophysiology, Vol. 131, No. 3, pp. 676-693, Mar. 2020, https://doi.org/10.1016/j.clinph.2019.11.013

[9] J. L. Herrero, S. Khuvis, E. Yeagle, M. Cerf, and A. D. Mehta, "Breathing above the brain stem: volitional control and attentional modulation in humans," Journal of Neurophysiology, Vol. 119, No. 1, pp. 145-159, Jan. 2018, https://doi.org/10.1152/jn.00551.2017

[10] A. D. Farmer, "Non-inasive vagus nerve stimulation-Hope or hype?," Neurogastroenterology and Motility, Vol. 32, No. 4, Apr. 2020, https://doi.org/10.1111/nmo.13822

[11] M. E. Bates, L. M. Lesnewich, S. G. Uhouse, S. Gohel, and J. F. Buckman, "Resonance-paced breathing alters neural response to visual cues: proof-of-concept for a neuroscience-informed adjunct to addiction treatments," Frontiers in Psychiatry, Vol. 10, Sep. 2019, https://doi.org/10.3389/fpsyt.2019.00624 
[12] R. McCraty and M. A. Zayas, "Cardiac coherence, self-regulation, autonomic stability, and psychosocial well-being," Frontiers in Psychology, Vol. 5, Sep. 2014, https://doi.org/10.3389/fpsyg.2014.01090

[13] "Building Personal Resilience", A handbook for HeartMath certified coachers and mentors, Institute of HeartMath, 2014.

[14] H.-G. Kim, E.-J. Cheon, D.-S. Bai, Y. H. Lee, and B.-H. Koo, "Stress and heart rate variability: a metaanalysis and review of the literature," Psychiatry Investigation, Vol. 15, No. 3, pp. 235-245, Mar. 2018, https://doi.org/10.30773/pi.2017.08.17

[15] P. Ratanasiripong, J. F. Park, N. Ratanasiripong, and D. Kathalae, "Stress and anxiety management in nursing students: biofeedback and mindfulness meditation," Journal of Nursing Education, Vol. 54, No. 9, pp. 520-524, Sep. 2015, https://doi.org/10.3928/01484834-20150814-07

[16] T. L. Zucker, K. W. Samuelson, F. Muench, M. A. Greenberg, and R. N. Gevirtz, "The effects of respiratory sinus arrhythmia biofeedback on heart rate variability and posttraumatic stress disorder symptoms: A pilot study," Applied Psychophysiology and Biofeedback, Vol. 34, No. 2, pp. 135-143, Jun. 2009, https://doi.org/10.1007/s10484-009-9085-2

[17] Y. Kotozaki et al., "Biofeedback-based training for stress management in daily hassles: an intervention study," Brain and Behavior, Vol. 4, No. 4, pp. 566-579, Jul. 2014, https://doi.org/10.1002/brb3.241

[18] P. Paine, J. Kishor, S. F. Worthen, L. J. Gregory, and Q. Aziz, "Exploring relationships for visceral and somatic pain with autonomic control and personality," Pain, Vol. 144, No. 3, pp. 236-244, Aug. 2009 , https://doi.org/10.1016/j.pain.2009.02.022

[19] P. Windthorst et al., "Heart rate variability biofeedback therapy and graded exercise training in management of chronic fatigue syndrome: An exploratory pilot study," Journal of Psychosomatic Research, Vol. 93, pp. 6-13, Feb. 2017, https://doi.org/10.1016/j.jpsychores.2016.11.014

[20] A. Dobbin, J. Dobbin, S. Ross, C. Graham, and M. Ford, "Randomised controlled trial of brief intervention with biofeedback and hypnotherapy in patients with refractory irritable bowel syndrome," The Journal of the Royal College of Physicians of Edinburgh, Vol. 43, No. 1, pp. 15-23, Mar. 2013, https://doi.org/10.4997/jrcpe.2013.104

[21] E. Sowder, R. Gevirtz, W. Shapiro, and C. Ebert, "Restoration of vagal tone: a possible mechanism for functional abdominal pain," Applied Psychophysiology and Biofeedback, Vol. 35, No. 3, pp. 199-206, Sep. 2010, https://doi.org/10.1007/s10484-010-9128-8

[22] Edwards D. J. and Edwards S. D., "Report on a psychological well-being and resilience programme for rugby players," African Journal for Physical Activity and Health Sciences, Vol. 23, pp. 25-42, 2016.

[23] McCraty Rollin, Atkinson Mike, Tomasino Dana, and Raymond Trevor Bradley, "The coherent heart heart-brain interactions, psychophysiological coherence, and the emergence of system-wide order," Integral Review, Vol. 5, No. 2, pp. 10-115, Dec. 2009.

[24] M. Dupee, T. Forneris, and P. Werthner, "Perceived outcomes of a biofeedback and neurofeedback training intervention for optimal performance: learning to enhance self-awareness and self-regulation with olympic athletes," The Sport Psychologist, Vol. 30, No. 4, pp. 339-349, Dec. 2016, https://doi.org/10.1123/tsp.2016-0028

[25] M. J. Stern, R. A. F. Guiles, and R. Gevirtz, "HRV biofeedback for pediatric irritable bowel syndrome and functional abdominal pain: a clinical replication series," Applied Psychophysiology and Biofeedback, Vol. 39, No. 3-4, pp. 287-291, Dec. 2014, https://doi.org/10.1007/s10484-014-9261-x

[26] Morris A. and Leach B., "A qualitative exploration of the lived experiences of patients before and after ileostomy creation as a result of surgical management for Crohn's disease," Ostomy/wound management, Vol. 63, No. 1, Jan. 2017.

[27] G. Rein, M. Atkinson, and R. McCraty, "The physiological and psychological effects of compassion and anger," Journal of Advancement in Medicine, Vol. 8, No. 2, pp. 87-105, Jan. 1995.

[28] R. McCraty and F. Shaffer, "Heart rate variability: new perspectives on physiological mechanisms, assessment of self-regulatory capacity, and health risk," Global Advances in Health and Medicine, Vol. 4, No. 1, pp. 46-61, Jan. 2015, https://doi.org/10.7453/gahmj.2014.073

[29] S. D. Edwards, "Effects of biofeedback training on physiological coherence, health and spirituality perceptions: sport and exercise psychology," African Journal for Physical Health Education, Recreation and Dance, Vol. 20, No. 21, pp. 500-510, 2014.

[30] S. Pellissier, C. Dantzer, F. Canini, N. Mathieu, and B. Bonaz, "Psychological adjustment and autonomic disturbances in inflammatory bowel diseases and irritable bowel syndrome," 
Psychoneuroendocrinology, Vol. 35, No. 5, pp. 653-662, Jun. 2010, https://doi.org/10.1016/j.psyneuen.2009.10.004

[31] Jonathan A. Smith, Paul Flowers, and Michael Larkin, Interpretative Phenomenological Analysis: Theory, Method and Research. London: SAGE Publications, 2009, pp. 40-55.

[32] S. Hale, S. Grogan, and S. Willott, "Male GPs' views on men seeking medical help: A qualitative study," British Journal of Health Psychology, Vol. 15, No. 4, pp. 697-713, Nov. 2010, https://doi.org/10.1348/135910709x479113

[33] L. Horgan, H. Richards, and A. Joy, "The young adult male's perception of life with inflammatory bowel disease and a stoma: a qualitative examination," Journal of Clinical Psychology in Medical Settings, Vol. 27, No. 3, pp. 560-571, Sep. 2020, https://doi.org/10.1007/s10880-019-09695-3

[34] K. J. Kemper, D. Powell, C. C. Helms, and D. B. Kim-Shapiro, "Loving-kindness meditation's effects on nitric oxide and perceived well-being: a pilot study in experienced and inexperienced meditators," Explore, Vol. 11, No. 1, pp. 32-39, Jan. 2015, https://doi.org/10.1016/j.explore.2014.10.002

[35] I. Dundas, P.-E. Binder, T. G. B. Hansen, and S. H. Stige, "Does a short self-compassion intervention for students increase healthy self-regulation? A randomized control trial," Scandinavian Journal of Psychology, Vol. 58, No. 5, pp. 443-450, Oct. 2017, https://doi.org/10.1111/sjop.12385

[36] Davies Nj, "Learning self-compassion. A tool for your diabetes management kit," Diabetes SelfManagement, Vol. 29, No. 4, 2012.

[37] A. L. Baldwin, "Case report: control of heart rate variability to cope with stress and pain after colectomy," Biofeedback, Vol. 46, No. 3, pp. 60-64, Sep. 2018, https://doi.org/10.5298/1081-593746.3.01

[38] E. Persson, "Experiences of Swedish men and women 6 to 12 weeks after ostomy surgery," Journal of WOCN, Vol. 29, No. 2, pp. 103-108, Mar. 2002, https://doi.org/10.1067/mjw.2002.122053

[39] L. Keefer, "Behavioural medicine and gastrointestinal disorders: the promise of positive psychology," Nature Reviews Gastroenterology and Hepatology, Vol. 15, No. 6, pp. 378-386, Jun. 2018, https://doi.org/10.1038/s41575-018-0001-1

[40] A. H. Kemp and D. S. Quintana, "The relationship between mental and physical health: insights from the study of heart rate variability," International Journal of Psychophysiology, Vol. 89, No. 3, pp. 288296, Sep. 2013, https://doi.org/10.1016/j.ijpsycho.2013.06.018

[41] Q. Liu, E. M. Wang, X. J. Yan, and S. L. Chen, "Autonomic functioning in irritable bowel syndrome measured by heart rate variability: A meta-analysis," Journal of Digestive Diseases, Vol. 14, No. 12, pp. 638-646, Dec. 2013, https://doi.org/10.1111/1751-2980.12092

[42] I. A. Mouzas et al., "Autonomic imbalance during the day in patients with inflammatory bowel disease in remission. Evidence from spectral analysis of heart rate variability over 24 hours," Digestive and Liver Disease, Vol. 34, No. 11, pp. 775-780, Nov. 2002, https://doi.org/10.1016/s15908658(02)80070-6

[43] Daniela Jelenova, Marie Ociskova, Jan Prasko, Martina Hunkova, and Vladimir Mihal, "Heart rate variability in children with inflammatory bowel diseases," Neuro Endocrinology Letters, Vol. 36, No. 1, Feb. 2015.

[44] K. Lorig, "Chronic disease self-management: from randomized trial to public policy," European Journal of Public Health, Vol. 20, pp. 6-7, 2010.

[45] J. C. Torrado, J. Gomez, and G. Montoro, "Emotional self-regulation of individuals with autism spectrum disorders: smartwatches for monitoring and interaction," Sensors, Vol. 17, No. 6, p. 1359, Jun. 2017, https://doi.org/10.3390/s17061359

[46] K. Mercer, M. Li, L. Giangregorio, C. Burns, and K. Grindrod, "Behavior change techniques present in wearable activity trackers: a critical analysis," JMIR mHealth and uHealth, Vol. 4, No. 2, p. e40, Apr. 2016, https://doi.org/10.2196/mhealth.4461

[47] M. Halland, G. Parthasarathy, A. E. Bharucha, and D. A. Katzka, "Diaphragmatic breathing for rumination syndrome: efficacy and mechanisms of action," Neurogastroenterology and Motility, Vol. 28, No. 3, pp. 384-391, Mar. 2016, https://doi.org/10.1111/nmo.12737

[48] L. Yardley, "Demonstrating the validity of qualitative research," The Journal of Positive Psychology, Vol. 12, No. 3, pp. 295-296, May 2017, https://doi.org/10.1080/17439760.2016.1262624

[49] H. Benson and M. Z. Klipper, The Relaxation Response. Avon, 1975.

[50] K. M. Cloninger and C. R. Cloninger, "The psychobiology of the path to a joyful life: implications for future research and practice," The Journal of Positive Psychology, Vol. 15, No. 1, pp. 74-83, Jan. 2020, https://doi.org/10.1080/17439760.2019.1685579 


\section{Appendix}

Interview schedule:

1) How did you find the biofeedback sessions?

2) What was good about the training programme?

3) What did you learn?

4) Did this intervention help you with your gastrointestinal symptoms?

5) How, in what ways?

6) What was the most helpful thing you learnt on the course?

7) Were you able to reach the "state of coherence"?

8) And if so how did it feel?

9) What have you learnt about ways of managing stress?

10) Is there anything you will do differently in the future because of taking part in the study? 